\title{
Burden of diseases in poor resource countries: meeting the challenges of combating HIV/AIDS, tuberculosis and malaria
}

\author{
F.S. MHALU \\ School of Medicine, Muhimbili University College of Health Sciences, \\ P.O. Box 65488, Dar es Salaam, Tanzania \\ E-mail:fmhalu@muchs.ac.tz
}

\begin{abstract}
Poverty, ill health and ignorance are closely interlinked and each is a determinant of the other. HIV/AIDS, malaria and tuberculosis are by far the commonest causes of ill-health and death in the poorest countries of the world which happen to be in the tropics and temperate countries in Africa, Asia and South America. Morbidity and mortality from these three diseases have a major socio-economic impact on individuals, communities and nations, due to the vicious cycle of poverty, ill health and ignorance. In Tanzania morbidity due to HIV/AIDS, tuberculosis and malaria leads to irrecoverable losses in productivity, inadequately trained workforce due to absence from training by the sick, heavy health care budgets to treat these otherwise preventable diseases, less competitive economy, higher labour force turnovers and unstable national budgets. If not controlled continuing rise in incidence of HIV/AIDS, malaria and TB may threaten the survival of small enterprises and ability to attract foreign investments leading to a rise in unemployment. Thus, investments in the improvement of health including HIV/AIDS, malaria and TB if done well will bring substantial benefits for the national economy including an increase in productivity. In this paper a review of the impact of HIV/AIDS, TB and malaria in Tanzania is done with an attempt to propose how research can contribute to improved efforts towards more effective prevention and control efforts. The need for multidisplinary research efforts in addressing the three disease conditions is proposed.
\end{abstract}

Key words: HIV/AIDS, malaria, tuberculosis, burden, poverty, research

\section{Introduction}

The eight Milennium Development Goals were derived from the Milennium Declaration which was signed by 189 countries (MDG, 2005). Globalization process by the world community includes HIV/AIDS, tuberculosis and malaria among the 8 goals. Poverty, ill health and ignorance are closely interlinked and each is a determinant of the other. HIV/AIDS, malaria and tuberculosis are by far the commonest causes of ill-health and death in the poorest countries of the world which happen to be in the tropics and temperate countries in Africa, Asia and South America. Illness and death from these three diseases have a major socioeconomic impact on individuals and communities due to the vicious cycle of poverty, ill health and ignorance.

HIV/AIDS, tuberculosis and malaria are among the top killers in the world. In Tanzania, ill-health resulting from HIV/AIDS, tuberculosis and other febrile illnesses including malaria leads to irrecoverable losses in productivity, inadequately trained workforce due to absence from training by the sick, heavy health care budgets to treat these otherwise preventable diseases, less competitive economy, higher labour force turnovers and unstable national budgets. If not controlled continuing rise in incidence of HIV/AIDS, malaria and tuberculosis may threaten the survival of small enterprises and ability to attract foreign investments leading to a rise in unemployment. Thus, investments in the improvement of health including HIV/AIDS, malaria and tuberculosis if done well will bring substantial benefits for the national economy including increase in productivity, a better trained workforce, improved economy, strong business enterprises, lower unemployment, fall in disease trends and better social well being of the population. It is therefore encouraging to note that most progressive businesses in Tanzania and elsewhere invest significantly towards health care of their workforces and their families. It is generally agreed that investing in health yields highest rates of return of between US\$3 and 10 for every one dollar invested compared to investment in many other sectors of development which yield at most US $\$ 1.5$ for every 1 invested dollar.

In this paper, the impact of HIV/AIDS, tuberculosis and malaria in Tanzania is reviewed. 
An attempt to propose how research can contribute to improved efforts towards more effective prevention and control efforts is made. It is suggested that there is need for multidisplinary research efforts in addressing the three disease conditions instead of current and past experience when each of the three diseases have been researched on in isolation.

\section{Millennium Development Goals and response to HIV/AIDS, Tuberculosis and Malaria}

Following the decision of the member states of the United Nations (UN) made in September 2000 and after consultations among international $\mathrm{UN}$ agencies including the World Bank, the International Monetary Fund, the Organization for Economic Cooperation and Development and the specialized agencies, the General Assembly of the UN came up with the Millennium Development Goals as part of the road map for creating a global partnership for development with the goals and targets which among other priorities address HIV/AIDS, tuberculosis and malaria. The Milennium Development Goals aim at eradicating extreme poverty and hunger; achieving universal primary education; promoting gender equality and empowering women; reducing child mortality; improving maternal health; combating HIV/AIDS, malaria, and other diseases; ensuring environmental sustainability; and developing a global partnership for development.

To date, in Tanzania many poor people, children and women in particular, die without ever accessing a health facility. Equitable and sustained access to care, support and treatment are essential to improve the well-being and life expectancy of people living with HIV/AIDS, but issues pertaining to finances, infrastructure, human, and logistical weaknesses need to be resolved first, so as not to further weaken an already constrained health system. All these call for well designed studies.

Trend in health service outputs during the 1990s in Tanzania show a mixed picture despite some successes in child immunisation coverage and in tuberculosis treatment. There exists a huge burden of disease across all age groups. Rural areas and the poor remain disadvantaged both in terms of outcomes and service uptake. It is thus important to ensure that health services are accessible to the poor population and the quality of services is improved. Also important is the need to strengthen disease surveillance and routine health management information systems to generate indicators for measuring service delivery and maintaining appropriate and effective data management for easy processing and dissemination (NSGRP, 2005).

According to Tanzanian National Strategy for Growth and Reduction of Poverty (NSGRP, 2005), survival indicators are not encouraging. No substantial progress was made in the reduction of infant and under-five mortality and maternal mortality. There are indications of slight increase in recent years, probably related to HIV/AIDS pandemic. HIV/AIDS pandemic has a wider implication for other indicators such as life expectancy. It is also important to note that high rate of infant and child mortality is due to continuing high prevalence of malaria and malnutrition. Also poor capacity in diagnosis and the emergence of antimicrobial drug resistance yet pose another challenge for policy and decision making. All these require valid supportive data and information to provide basis for effective interventions. Thus, health research remains an important tool for national development.

\section{Burden of HIV/AIDS, Tuberculosis and Malaria in Tanzania}

The burden of disease in Tanzania is due predominantly to potentially preventable diseases including HIV/AIDS, malaria, tuberculosis, and childhood and reproductive conditions. Data on some health interventions are more hopeful. For example, use of insecticide treated bednets has risen; and coverage of effective tuberculosis treatment has expanded.

The increasing prevalence of HIV/AIDS is considered to be the most significant threat to socioeconomic development in the country, and tuberculosis incidence has risen concomitantly, with an estimated $40 \%$ of new tuberculosis cases in recent years testing positive for HIV (Ministry of Health, unpublished). In addition, malaria is a leading cause of death among children, and its role in anaemia among pregnant women is substantial (MOH, 2003). The leading causes of death in 
Tanzania are malaria (22\%), AIDS (17\%), tuberculosis $(9 \%)$, pneumonia $(6.5 \%)$, and anemia (5.5\%). Clearly, communicable diseases exact a heavy toll throughout the country and in all age groups (Ministry of Health, unpublished).

HIV/AIDS is by far the leading cause of premature mortality in sub-Saharan Africa and the fourth-biggest killer worldwide. At the end of 2004, an estimated 39 million people globally were living with HIV. There were 3.1 million AIDS deaths in 2004, including 510,000 child deaths. In subSaharan Africa, HIV prevalence rates among adults have reached around $7.4 \%$, rising to over $20 \%$ in some settings. Globally, just under half the people living with HIV are female, but as the epidemic worsens, the share of infected women and girls is growing.

The estimated prevalence of HIV in Tanzania is approximately $12 \%$ in adults aged $15-49$ (UNAIDS, 2001) and approximately 2 million persons are living with HIV/AIDS in the country (out of a population of 34.5 million). The epidemic has become generalized throughout the country, with certain geographic areas and population groups having higher disease prevalence. AIDS is now thought to be the leading cause of death among adults (MEASURE, 2001).

Estimates of the number of acute malaria cases are highly variable, and range up to 500 million. At a minimum, 1 million people die from malaria every year, and malaria is likely to be a contributing factor in another 2 million deaths. About $80 \%$ of malaria deaths are among young children living in subSaharan Africa. Malaria mortalilty among children 0-4 years in sub-Saharan Africa in the year 2002 was estimated at more than 800,000 deaths. Today, $40 \%$ of the world's population - primarily those living in the world's poorest countries - are at risk of contracting malaria. In many parts of Africa, children experience at least three life-threatening infections by the age of one; those who survive may suffer learning impairments or brain damage. Pregnant women and their unborn children are also at particular risk of malaria, which is a cause of perinatal mortality, low birth weight and maternal anaemia (WHO, 2005).

Malaria is the leading cause of death among children and adults in Tanzania, and is also the leading cause of in-patient and outpatient health service attendance in all ages (Kitua, 2003). Eighty percent of the country is highly endemic for the disease, and $90 \%$ of the population is at risk. As a result, malaria is a major cause of socioeconomic problems in the country, due to both direct treatment costs and indirect costs such as lost income and lost productivity. Development of resistance to antimalarial drugs is a growing problem and requires continued vigilance to ensure that appropriate and technically feasible strategies are implemented to combat the disease.

Tuberculosis kills nearly 1.7 million people annually, most of them in their prime producutive years (WHO, 2005). The emergence of drugresistant strains of the disease, the spread of HIV/AIDS, which enhances susceptibility to tuberculosis, and the growing number of refugees and displaced persons, have all contributed to its spread. In 2003, there were an estimated 8.8 million new cases, including 674,000 in people infected with HIV. The number of new tuberculosis cases has been growing by about $1 \%$ a year, predominantly because of AIDS epidemic in subSaharan Africa.

Similarly, the burden of tuberculosis infection and active tuberculosis disease has rapidly increased in Tanzania, due primarily to the HIV epidemic; it is believed that approximately $50 \%$ of the population is infected with tuberculosis. Tuberculosis prevalence increased five-fold from 11,700 cases in 1983 to 61,000 cases in 2001. Tuberculosis, the leading opportunistic infection among HIV-positive persons, is also a leading cause of death in this population; tuberculosis infection is more likely to progress to active tuberculosis disease among those who are HIV-positive. Tuberculosis mortality has increased to approximately $10 \%$ due to the high prevalence of HIV co-infection in Tanzania, and tuberculosis is a leading cause of morbidity. Efforts are underway to begin to coordinate HIV/AIDS and tuberculosis programme by sharing resources and using Voluntary Counselling Testing for HIV as an entry point for tuberculosis screening.

\section{Targets and indicators on the HIV/AIDS, Tuberculosis and Malaria}

Two targets have been set on the three diseases. 
Target 1 requires to halt the continuing spread by 2015 and reverse the spread of HIV/AIDS. The MDG indicator on HIV prevalence among young pregnant women is used as an indicator of the new infection rate in population (WHO, 2005). Currently, not enough data are available to provide full trend analysis for this indicator. Worst still, there are still few countries that have collected data on condom use at last sex with a non-cohabiting partner. Data available in $39.6 \%$ of countries in subSaharan Africa indicate that only $23 \%$ and $41 \%$ of young men and women, respectively, report using a condom at last sex with a non-cohabiting partner. On the other hand, target 2 requires to halt by 2015 and reverse the incidence of malaria, tuberculosis and other major diseases.

The success of Directly Observed Treatment Short-course (DOTS) depends on expanding case detection while ensuring high treatment success rates. The global treatment success rate for DOTS programmes was $82 \%$ for the cohort of patients registered in 2002. However, cure rate tend to be lower, and death rates higher, where drug resistance is frequent, or HIV prevalence is high.

\section{Relevance of the Millenium Development Goal to Tanzania}

HIV/AIDS, acute febrile illness (malaria), tuberculosis and other infective agents account for up to $70 \%$ of causes of death in Ilala, Hai and Morogoro districts and it is likely that other parts of Tanzania are going through the same pattern (AMMP, 1997). In fact for those of us who have recently been attending burials of relatives, neighbours and workmates, it does not need an expert to inform us which are the major causes of ill health and death; it is HIV/AIDS, tuberculosis and febrile illness. The millennium development goals are highly relevant to Tanzania and we should commend our Government for endorsing the strategy and for coming up with the Poverty Reduction Strategy Paper beginning 2000. It is therefore essential for the health research institutions and their scientists to come up with strategies and initiate activities on how best to respond to the challenges preventing fast reduction of ill health and death brought about by the three diseases.
To date, many poor people, children and women in particular, die without ever accessing a health facility. Equitable and sustained access to care, support and treatment are essential to improve the well-being and life expectancy of people living with HIV/AIDS, but issues pertaining to finances, infrastructure, human, and logistical weaknesses need to be resolved first, so as not to further weaken an already constrained health system. All these call for well designed studies.

In doing so it will be necessary to have joint efforts addressing all the three diseases together because all of them lead to fever which is often taken as malaria for the reason that malaria is easily managed by affected individuals at home under the primary health care approach while tuberculosis and HIV/AIDS require professional health expertise. Among adults living in malaria endemic areas, presentation of HIV associated conditions including tuberculosis, salmonelosis other than due to Salmonela typhi and other bacterial and fungal causes of septicaemia are the leading aetiologies of fever of more than 2 weeks duration while malaria is an insignificant finding. Moreover, for those with fever and blood stream microbial infections, one study in Dar es Salaam found that $81 \%$ had HIV infection (Archibold et al., 1998). It is also known that among under five years old children with fever and or pallor in malaria endemic areas, only between $38 \%$ and $70 \%$ are proven to have laboratory evidence of malaria (Nsimba et al., 2002; Tarimo et al., 2001) thus there is a need to make intensive research efforts and advocacy into availing simple, accurate rapid and affordable diagnostic tests for malaria and consider to include investigations for HIV and tuberculosis for adults and children with febrile episodes.

Regarding tuberculosis, it is estimated that life time risk of acquisition of active tuberculosis in Tanzania is $10 \%$ for HIV free individuals and $50 \%$ for those with HIV with an overall annual incidence of about $1 \%$ for all forms of tuberculosis and $0.5 \%$ for smear positive tuberculosis.

Moreover it is estimated that more than $50 \%$ of all Tanzanian adults are living with quiescent Mycobacterium tuberculosis in their lungs which can flare up whenever immunosuppression sets in. It is therefore clear that more accurate and cost effective simple/rapid methods for detection of 
tuberculosis are required instead of relying on microscopy alone in current use which has less than optimal sensitivity. It is encouraging to note that improved serological tests for tuberculosis are emerging.

\section{HIV/AIDS, malaria and tuberculosis in relation to Poverty}

While more than $95 \%$ of new cases of HIV/AIDS, malaria and tuberculosis in the world are occurring in developing countries including Tanzania, and while poverty is clearly associated with malaria and tuberculosis due to poor housing and environmental sanitation, there is no evidence yet to indicate that poverty within Tanzania is a risk factor for HIV/AIDS. On the contrary increased socioeconomic status of a few individuals living among the majority of the population with low incomes may increase HIV risk for the socio-economically better off compared with the poor majority. Further studies on the relationship of HIV/AIDS and socio economic status of individuals and their sex partners are therefore required inorder to sharpen the current intervention measures against the HIV/AIDS crisis.

The socio-economic burdens of tuberculosis, malaria and HIV/AIDS on communities are huge, thus it has been estimated that at the individual level, tuberculosis costs up to four months of lost income to the individual amounting to up to $30 \%$ of annual household income while malaria has been estimated to reduce economic growth in Africa by $1.3 \%$ while when HIV/AIDS reaches a prevalence rate of $8 \%$ or more as is the case in many urban and rural communities in Tanzania, economic cost in growth is about $1 \%$ or more per a year (Guwatudde et al., 2003; Wojcicki et al., 2005).

\section{Proposed strategies to be adopted by research and development institutions}

In fullfilment of the MDGs and Tanzania's Poverty Reduction Strategy, health research and development (R\&D) institutions in Tanzania should strategize and plan on how research in their institutions can contribute to reduction of poverty in the remaining period before 2015. In this regard R\&D institutions may wish to come up with clear and specific research goals, strategies and activities. Since, for instance, the National Institute for
Medical Research (NIMR) already has Centres dedicated to malaria and tuberculosis research, the institution may wish to consider the establishment of an HIV/AIDS research programme at one or more Centers which will concentrate on biomedical, clinical, epidemiology, public health and social behavioural studies in HIV/AIDS and related conditions. NIMR should also encourage the Centers currently dealing with malaria and tuberculosis research to dedicate most of their research efforts in the coming few years to address the priority research needs towards poverty reduction.

Among priority research areas which could be undertaken include: developing and evaluating simple/rapid diagnostic assays for malaria, tuberculosis and HIV infection to ensure costeffective treatment and monitoring of trends of the diseases; determining continuing role of poverty and illiteracy in trends of HIV/AIDS, malaria and tuberculosis; developing and evaluating safety, immunogenicity and efficacy of appropriate HIV, tuberculosis and malaria vaccines and vaginal microbicides against HIV and other sexually transmitted infections; drug development and conducting clinical trials of therapies to manage HIV/AIDS, tuberculosis and malaria; improving clinical management of HIV, malaria and tuberculosis including associated conditions; preventing mother to child HIV transmission and involving their male partners, monitoring and evaluating the implementation of the research programme on HIV, tuberculosis and malaria towards achieving the millennium development goals; and improvement of environmental sanitation against tuberculosis and malaria.

\section{Conclusion}

The Milennium Deveopment Goals provide a common set of priorities for addressing poverty. Health is the heart of these goals. It is recognised that health is central to global agenda of reducing poverty as well as an important indicator of human well-being. Health is represented in three of the eight goals, and makes an acknowledged contribution to the achievement of all the other goals, in particular those related to the eradication of extreme poverty and hunger, education, and gender 
equality, importantly, the health goals also focus on problems which disproportionally affect the poor (WHO, 2005).

In 2005 we are slightly more than halfway towards the MDG target date of 2015 (targets are set against 1990 baselines). Overall, the data available suggest that if trends observed during the 1990s continue, the majority of poor countries will not meet the health MDGs (WHO, 2005). For instance, none of the poorest regions of the developing world is currently on tract to meet the child mortality target.

Tackling poverty-related diseases and conditions is central to global efforts in reducing the gaps between haves and have-nots. Efforts to achieve the MDGs should therefore be part of research institutions core strategies. However, our partners should strive towards assisting developing countries in their efforts to tackle HIV/AIDS, tuberculosis and malaria.

It is time that our research institutions and their partners mark the beginning of efforts in working towards the MDGs in the area of HIV, tuberculosis and malaria. In working towards achieving the national poverty reduction strategy, the Government of Tanzania should consider the necessity of setting aside at least $2 \%$ of poverty reduction strategy funds to support research which is essential to provide the technology, skills and verifiable information.

\section{References}

AMMP (1997) Adult Morbidity and Mortality Project Report. Ministry of Health, Tanzania.

Archibold, L.K., den Dulk, M.O., Pallangyo, K.J., Reller, L.B. (1998) Fatal Mycobacteium tuberculosis blood stream infections in febrile hospitalized adults in Dar es Salaam, Tanzania. Clinical Infectious Disease 26, 290-296.
Guwatudde, D., Zalwango, S., Kamya, M.R., Debanne, S.M., Diaz, M.I., Okwera, A., Mugerwa, R. D., King, C. \& Whalen, C.C. (2003) Burden of tuberculosis in Kampala Uganda. Bulletin of the World Health Organization 81, 799-805.

Kitua, A.Y. (2003) Malaria control in the context of integrated management of childhood illness in Tanzania: the challenges ahead. Tanzania Health Research Bulletin 5, 1-4.

MDG (2005) Millenium Development Goals: About the Goals. The World Bank http://www.developmentgoals.org

MEASURE (2001) AIDS in Africa during the nineties: Tanzania.

MoH (1990) Health Policy. Ministry of Health, Dar es Salaam, Tanzania.

MoH (2003) National Malaria Control Programme in Tanzania. National Medium-Term Strategic Plan, 2003-2007, Ministry of Health, Dar es Salaam, Tanzania.

Nsimba, S.E.D., Massele, A.Y., Eriksen, J., Gustasson, L.L., Tomson, G. \& Warsame, M. (2002) Case management of malaria in under-fives at primary health care facilities in a Tanzania district. Tropical Medicine and International Health 7, 201-209.

Tarimo, D.S., Minjas, J.N. \& Bygbjerg, I.C. (2001) Malaria diagnosis and treatment under the strategy of the integrated management of childbood illness (IMCI): relevance of laboratory support from the rapid immunochromatic tests of ICT Malaria $\mathrm{Pf} / \mathrm{Pv}$ and OptiMal. Annals of Tropical Medicine \& Parasitology 95, 437-444.

WHO (2005) Health and the Milennium Development Goals. World Health Organization, Geneva.

Wojcicki, J.M. (2005) Socio-economic status as a risk factor for HIV infection in women in East, Central and Southern Africa: a systematic review. Journal of Biosocial Science 37, 1-36. 